\title{
The definition of basic parameters of the set of small-sized equipment for preparation of dry mortar for various applications
}

\author{
Inga Emelyanova ${ }^{1}$, Vladimir Blazhko, ${ }^{1, *}$, Svetlana Dansheva ${ }^{1}$, and Natalia Shyshko ${ }^{1}$ \\ ${ }^{1}$ Kharkiv National University of Civil Engineering and Architecture, Department of Mechanization of \\ Construction Processes, Sumska st. 40, 61002 Kharkiv, Ukraine
}

\begin{abstract}
Based on the conducted information retrieval and review of the scientific literature, unsolved issues have been identified in the process of preparation of dry construction mixtures in the conditions of a construction site. The constructions of existing technological complexes for the production of dry construction mixtures are considered and their main drawbacks are identified in terms of application in the conditions of the construction site. On the basis of the conducted research, the designs of technological sets of small-sized equipment for the preparation of dry construction mixtures in the construction site are proposed. It is found out that the basis for creating the proposed technological kits are new designs of concrete mixers operating in cascade mode. A technique for calculating the main parameters of technological sets of equipment is proposed, depending on the use of the base machine of the kit.
\end{abstract}

\section{Introduction}

Modern dry building mixtures are complex compositions of components, different in their properties. Mixing these components together and creating a homogeneous mixture is a difficult task in a technical sense. Creation of effective equipment for these purposes is an urgent problem of modern construction. With its solution, special attention should be paid to the development of technological kits.

At present, in the process of preparation of dry construction mixtures, there is a number of unresolved issues: increased wear of the working bodies and mixer bodies, long time for the preparation of complex compositions, the presence of segregation during the mixture preparation and the complexity of preparing the mixtures directly at the construction site [14].

At present, technological complexes, which have the ability to produce mixtures of different types, are used for the preparation of dry construction mixtures [5-7].

The complexes are equipped with high-quality dosing devices and forced-action mixers with one or two shafts, on which various stirring devices are fixed [3, 9]. However, modern concrete mixers do not always allow to produce homogeneous mixtures with a small filling factor of the working volume of the machine. In addition, existing mixers do not allow to

* Corresponding author: blagko-2008@ukr.net 
create a single organized work cycle in one place. From these positions, technological kits that allow to combine all operations in time are more attractive.

In addition, the variety of existing equipment, as well as technological lines and kits, is not aimed at solving the problem of making dry construction mixtures directly on the construction site.

Creation of effective small-sized equipment for the preparation of dry mixtures is an urgent task. The results of laboratory and industrial tests of new design mixers operating in a cascade mode, confirm their effectiveness in the preparation of dry building mixtures, which makes it possible to equip these machines with technological kits and complexes [8].

Main material and results. It is proposed to develop and introduce small-size sets of equipment for the new mixers operating in a cascade mode in the production of small amounts of work (with individual low-rise construction, construction in the agricultural sector, repair and reconstruction of existing buildings). Technological equipment kits are shown in Fig. 1. All equipment of these technological kits is based on a common base and is selected according to the performance of the base machine-mixer.

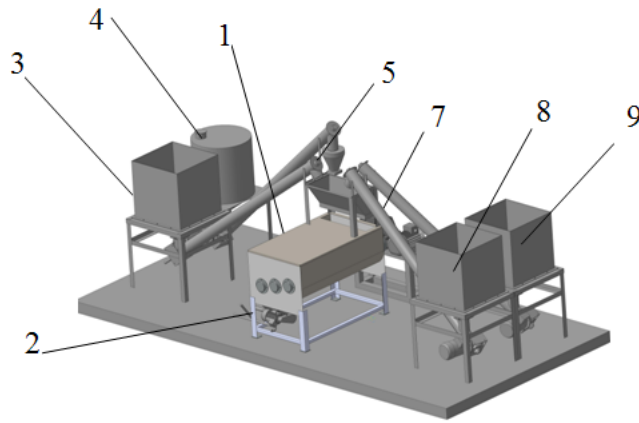

(a)

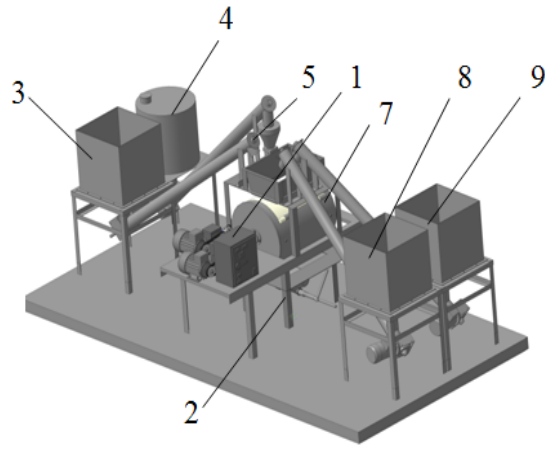

(b)

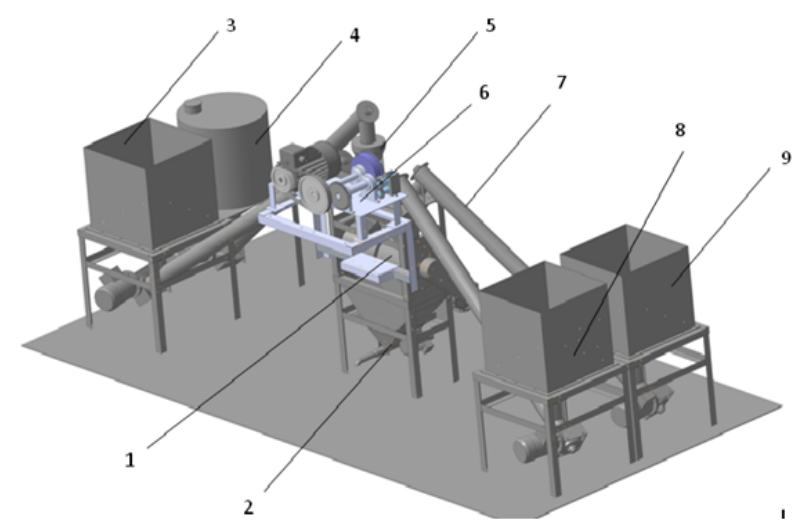

(c)

Fig. 1. Technological sets of equipment for the preparation of dry building mixtures: (a) the base machine is a three-shaft mixer; (b) the base machine is a mixer of gravitational-forced action; (c) base machine - two-rotor turbulent mixer

The technological set of equipment, presented in Fig. 1, includes the following components: 1 - mixer (basic machine); 2 - filling machine 3 - sand bunker; 4 - silo of cement; 5 - weighing batcher; 6 - fiber cutter; 7 - auger feeder; 8 - bunker of granite grains; 9 - filler hopper.

The basic machines of the technological set (Fig. 1) can be concrete mixers of a new constructive solution: a three-shaft concrete mixer, gravity-forced concrete mixer, a two- 
side turbulent mixer [5]. A feature of the work of these machines is the creation of a complex multi-path motion of the particles of the mixture in the body of the machine [9]. The adjacent equipment of the technological set is unified and has a traditional design solution.

\section{Theoretical part}

The productivity of a technological equipment set is determined on the basis of the technical performance of the base machine used in the composition of the technological set.

The productivity of the technological set with the use of a three-shaft concrete mixer is found according to the dependence [10]

$$
P_{\text {tech }}=3600 \cdot \frac{\pi}{4} \cdot\left(D^{2}-d^{2}\right) \cdot b \cdot n \cdot z_{b} \cdot \operatorname{Sin} \alpha \cdot k_{l}^{\text {aver }} \cdot k_{r}^{\mathrm{II}},
$$

where $D$ - is the diameter of the shaft along the end of the blade, m;

$d$ - is the diameter of the average shaft, $\mathrm{m}$;

$b$ - blade width, m;

$Z_{b}-$ there is number of blades of the middle shaft;

$\alpha-$ is the angle of installation of the blades, deg;

$k_{l}^{a v}-$ load factor of the mixer relative to the average shaft, $k_{l}=0.75$;

$n$ - frequency of rotation of the shaft of the working element;

$k_{r}^{I I}$ - coefficient of return of the concrete mixture of the second zone.

The technical performance of a set of equipment can be defined as

$$
P_{\text {tech }}=V_{o v} \cdot Z_{l}=\frac{1}{2}\left[\pi \cdot L_{b}\left(R_{b}^{2} \cdot k-r_{s h}^{2}\right)-\pi \cdot r_{n}^{2} \cdot l_{b} \cdot z_{l}-z_{1} \cdot b_{1} \cdot h_{1} \cdot c_{1}-z_{2} \cdot b_{2} \cdot h_{2} \cdot c_{2}\right] \cdot Z_{c},
$$

where $V_{o v}-$ is the total volume of the mixture in the mixer, $\mathrm{m}^{3}$;

$Z_{c}=3600 / t_{c}$ - there is number of cycles of machine operation per hour $\left(\mathrm{t}_{\mathrm{c}}-\right.$ is the duration of one cycle, which consists of the sum of time for loading components $t_{1}$, mixing them $t_{2}$ and unloading the finished mixture $t_{3}\left(t_{c}=t_{1}+t_{2}+t_{3}\right)$, s);

$L_{b}$ - length of the mixer body, m;

$R_{b}-$ is the radius of the mixer body, $\mathrm{m}$;

$k-$ is a coefficient that takes into account the position of the mixture in the body;

$R_{b}, l_{b}, z_{b}, r_{s h}$ - radius, length, number of legs of shaft blades and shaft radius, m;

$b_{1}, b_{2}, h_{1}, h_{2}, c_{1}, c_{2}$ - length, height and thickness of blades of the body and blades of the mixer shaft, $\mathrm{m}$.

The productivity of the technological set when using a two-rotor turbulent mixer is found according to the dependence

$$
P_{\text {tech }}=V_{o v} \cdot Z_{b} \cdot k_{m},
$$

where is $V_{o v}$ - the total volume of the mixture in the mixer, $\mathrm{m}^{3}$;

$Z_{b}=3600 / t_{b}$ - there is number of cycles of machine operation per hour $\left(t_{b}\right.$ is the duration of one cycle, which consists of the sum of the time for loading components $t_{1}$, mixing them $t_{2}$ and unloading the finished mixture $\left.\mathrm{t}_{3}\left(t_{b}=\mathrm{t}_{1}+\mathrm{t}_{2}+\mathrm{t}_{3}\right), \mathrm{s}\right)$;

$k_{m}$ - is the machine utilization factor $(0.85)$.

In this case, the feeding of the components of the concrete mixture into the mixer will be as follows:

$$
M=\int_{0}^{t}\left(Q x_{e n t}-Q x_{e x}\right) d t,
$$


where $M$ - total mass of components;

$t$-the time interval for loading all components;

$Q x_{e n t}$ - the flow of the components of the mixture upon leaving the storage bin;

$Q x_{e x}$ - the flow of the components of the mixture as it leaves the feeder.

The ratio of the total mass of $M$ components to the entire working volume of mixer $V$ is as follows:

$$
\frac{M}{V}=\Delta x=\frac{Q}{V} \int_{0}^{t}\left(x_{e n t}-x_{e x}\right) d t,
$$

where $x_{e n t}, x_{e x}, x$-respectively, the content of the components at the inlet, outlet and inside the mixer;

$Q$ - the capacity of the mixer under steady operating conditions.

Among the main performance indicators of a small-sized technological set of equipment for the preparation of a building mixture, it is worth mentioning the power costs.

The power costs for the operation of a technological equipment set intended for the preparation of fiber-concrete mixtures consist of the sum of the power costs of the individual types of equipment that are included in the kit (Fig. 1c): the machine-tool of the fiber, the base machine (concrete mixer), feeders and dispensers. Thus, the dependence for determining the power of the technological set of equipment has the form [7]

$$
N_{t . c}=N_{p . c}+N_{p}+N_{f}+N_{d},
$$

where $N_{t . c}$ - Total costs of power for the operation of the technological set of equipment, $\mathrm{kW}$;

$N_{p . c}$ - power costs of the base machine, $\mathrm{kW}$;

$N_{p}$ - power consumption of a fiber cutter, $\mathrm{kW}$;

$N_{f}$ - power consumption of feeders, $\mathrm{kW}$;

$N_{d}$ - power consumption of dispensers, $\mathrm{kW}$.

Taking into account the design features of the base machine of the three-shaft concrete mixer (Fig. 1), when working on dry building mixtures, $N_{t . c}$ should be defined as for a three-shaft mixer $N_{b . m}$, taking into account the operation of the machine on dry building mixtures

$$
N_{t . c}=\frac{N_{1}+N_{2}+N_{3}}{1000 \cdot \eta},
$$

where $N_{l}$ - the power used to mix the components of the mixture with the upper shaft;

$N_{2}$ - the power used to mix the components of the concrete mix and transport it to the discharge port by the middle shaft;

$N_{3}$ - the power used to mix the components of the mixture by the lower shaft;

$\eta$ - the efficiency of the mixer drive.

$$
N_{1}=N_{1}^{\prime}+N_{1}^{\prime \prime}
$$

where $N_{l}^{\prime}$ - the power expended on the mixing process in the first zone,

$N_{l}^{\prime \prime}$ - power costs for the mixing process in the second zone

$$
N_{1}^{\prime}=\omega \cdot F_{b} \cdot C_{1} \cdot f \cdot\left(R^{2}-r^{2}\right) \cdot Z_{b}^{I} \cdot k_{l}^{u p} \cdot \rho_{1},
$$

where $\omega$ - angular velocity of the shaft, $\mathrm{s}^{-1}$;

$F_{b}$ - blade area, $\mathrm{m}^{2}$;

$C_{l}$ - coefficient of resistance to movement of particles of dry concrete mixture along the blades in the first zone, $C_{l}=9$;

$f$ - coefficient of friction of the dry mixture over the surface of the blades, $f=0,4$; 
$R$ - outer radius of rotation of the blade, $\mathrm{m}$;

$r$ - internal radius of rotation of the blade, $\mathrm{m}$;

$Z_{b}^{I}$ - there is number of blades on the upper shaft in the first zone;

$k_{l}^{u p}$ - load factor of the concrete mixer relative to the upper shaft;

$\rho_{l}-$ average density of dry mix, $\mathrm{kg} / \mathrm{m}^{3}$.

$$
N_{1}^{\prime \prime}=\omega \cdot F_{b} \cdot C_{2} \cdot f^{\prime} \cdot\left(R^{2}-r^{2}\right) \cdot Z^{\mathrm{II}}{ }_{b} \cdot k_{l}^{u p} \cdot \rho_{2},
$$

where $C_{2}$ - coefficient of resistance to movement of particles of the dry mixture along the blades in the second zone, $C_{2}=7$;

$f^{\prime}-$ coefficient of friction of the mixture along the blade surface, $f^{\prime}=0.1 \ldots 0.2$;

$Z^{I I}{ }_{b}$ - there is number of blades on the upper shaft in the first zone.

After the transformations, the dependence for determining the power costs by the upper shaft of the mixer has the form

$$
N_{1}=\omega \cdot F_{b} \cdot \rho \cdot\left(R^{2}-r^{2}\right) \cdot k_{3} \cdot C_{1} \cdot f \cdot Z_{b}+C_{2} \cdot f^{\prime} \cdot Z_{b}^{\prime},
$$

The power expended on mixing the components of a concrete mix and its transportation to the discharge port by an average shaft

$$
N_{3}=N_{2}^{\prime}+N_{2}^{\prime \prime},
$$

where $N_{2}^{\prime}$ - the power expended on the process of mixing the components of the dry building mixture in the first zone and its transportation to the second zone;

$N_{2}^{\prime \prime}$ - power expended in the second zone of the mixer.

$$
N_{2}^{\prime}=m_{c} \cdot g \cdot W \cdot \omega \cdot R_{s},
$$

where $m_{c}$ - mass of the components of the mixture in the auger zone, $\mathrm{kg}$;

$W$ - Drag coefficient (when the body moves in hostile environments $W=4$ );

$\omega$ - Angular speed of rotation of the shaft, s;

$R_{s}$ - Radius of screw, m;

$g-$ acceleration of gravity

$$
N_{2}^{\prime \prime}=\omega \cdot F_{b} \cdot C_{2} \cdot f^{\prime} \cdot\left(R^{2}-r^{2}\right) \cdot Z_{b}^{I I} \cdot k_{l}^{u p} \cdot \rho_{2},
$$

where $Z_{b}^{I I}$ - there is number of blades on the middle shaft in the second zone.

The power required to mix the components of the mixture with the lower shaft

$$
N_{3}=N_{3}^{\prime}+N_{3}^{\prime \prime},
$$

where $N_{3}^{\prime}$ - power expended on the process of mixing in the first zone;

$N_{3} / \prime$ - the power expended on the mixing process in the second zone of the mixer;

$N_{3}^{\prime}$ and $N_{3}^{\prime \prime}$ - are determined by analogy with the upper shaft.

For a gravity-forced-action mixer [5] $N_{t, c}$, spent on the process of preparing a dry construction mix, consists of the power required to rotate the mixer body and the power required to rotate the blade shaft

$$
N_{t . c}=N_{b}+N_{s},
$$

where $N_{b}$ - power required to rotate the body of the mixer,

$$
N_{b}=N_{s}^{(1)}+N_{s}^{(2)}=\frac{0.85 \cdot G_{c, m} \cdot h \cdot Z \cdot \omega_{b}}{\eta_{d} \cdot 1000}+\frac{F_{f r} \cdot V_{a, v} \cdot z_{1}}{\eta_{d} \cdot 1000}
$$

where $G_{c . m}$ - weight of the components of the mixture, N;

$h$ - vertical coordinate of mass mixture displacement in the body, m; 
$Z$ - there is number of mixes circulating in the body of the machine;

$\omega_{\kappa}-$ angular speed of rotation of the mixer body, $\mathrm{s}^{-1}$;

$\eta_{d}$ - efficiency of the machine drive;

$F_{f r}-$ the frictional force arising when the particles of the mixture move along the surface of the blade of the mixer body, $\mathrm{N}$;

$V_{a . v}$ - absolute velocity of the mixture particles along the body blades, $\mathrm{m} / \mathrm{s}$;

$z_{2}$ - number of blades on the inner surface of the body.

The power expended on actuating the working member of the machine

$$
N_{s h}=N_{b}^{(1)}+N_{b}^{(2)}=\frac{\omega_{s h} \cdot M_{s h}}{\eta_{d} \cdot 1000}+\frac{F_{f r} \cdot V_{a . v} \cdot z_{2}}{1000 \cdot \eta_{d}}
$$

where $\omega_{s h}$-angular speed of rotation of the mixer's blade shaft, $\mathrm{s}^{-1}$;

$M_{s h}$ - torque of the blade shaft, N·m;

$\eta_{d}$ - efficiency of shaft drive;

$F_{f r}$ - the friction force that occurs when a particle of a concrete mix moves along the blade surface, $\mathrm{N}$;

$V_{a . v}-$ absolute velocity of the mixture particle along the shaft blade, $\mathrm{m} / \mathrm{s}$;

$z_{2}-$ there is number of blades on the shaft.

When installing a two-rotor turbulent mixer

$$
N_{\text {set }}=\omega_{b . s} \cdot F_{b} \cdot q_{\text {mix. }} \cdot f \cdot(R-r) \cdot \operatorname{Cos} \beta \cdot Z_{b} \cdot k_{l} \cdot \rho_{1},
$$

where $\omega_{b . s}-$ angular speed of rotation of the mixer's blade shaft, $\mathrm{s}^{-1}$;

$F_{b}$ - the area of the blade, $\mathrm{m}^{2}$;

$q_{\text {mix }}$ - pressure on the blade from the mixture side, $\mathrm{Pa}$;

$f$ - coefficient of friction of the components of the mixture along the surface of the blades of the working element of the mixer of construction mixtures $f=0.4$;

$R$ - outer radius of blade rotation, $\mathrm{m}$;

$r$ - internal radius of blade rotation, $\mathrm{m}$;

$\mathrm{Z}_{b}$ - there is number of blades on the upper shaft in the first zone;

$k_{l}-$ load factor of the concrete mixer;

$\rho_{1}$ - average density of dry mix, $\mathrm{kg} / \mathrm{m}^{3}$;

$\beta$ - blade angle.

Thus, the proposed technological kits of small-sized equipment can be successfully used to work directly on the construction site.

\section{Conclusions}

1. Technological sets of small-sized equipment of a new configuration and a new design solution are proposed to perform small volumes of work in the conditions of a construction site.

2. Dependencies are given allowing to determine the performance indicators of new technological equipment sets for the preparation of dry building mixtures on the construction site.

\section{References}

1. I.A. Emelyanova, A.N. Baranov, V.V. Blazhko, Materials of the International Scientific and Technical Conference "Interstroymech-2005", 1, 38-43 (2005) 
2. I.A. Emelyanova, V.V. Blazhko, A.I. Anishchenko, Naukovii visnik budivnitsva, 52, 317-325 (2009)

3. A. Kudrolli, Reports on progress in physics, 67 (3), 209-247 (2004)

4. C.S. Campbell, Annual Revue of Fluid Mechanics, 22, 57-92 (1990)

5. I.A. Emelyanova, V.V. Blazhko, O.V. Dobrokhodova, A.I. Anishchenko, Materials of the International Scientific and Technical Conference "Interstroymeh-2009", 398-403 (2009)

6. I.A. Emelyanova, V.V. Blazhko, O.V. Dobrokhodova, A.I. Anishchenko, Proceedings «HEAVY MACHINERY - HV 2011», 11-14 (2011)

7. I.A. Emelyanova, V.V. Blazhko, O.V. Dobrokhodova, A.I. Anishchenko, Proceedings «HEAVY MACHINERY - HV 2014», p. 35 - 38 (2014)

8. I. Emel'yanova, A. Anishchenko, S. Evel, Concrete-mixers working in cascade mode: monograph (Tim Public Library, Kharkov, 2012)

9. I.A. Emelyanova, A.I. Anishchenko, F.A. Stoyanov, New materials and technologies in engineering: a collection of scientific papers on the results of the International Science and Technology Conference, 16, 137-140 (2012)

10. I.A. Emelyanova, V.V. Blazhko, A.S. Neporozhnev, V. Yu. Shevchenko, New materials and technologies in machine building: a collection of scientific Works on the results of the International Scientific and Technical Conference, 13, 155-157 (2011) 